\title{
Defective NOTCH signalling drives smooth muscle cell death and differentiation in bicuspid aortic valve aortopathy
}

\author{
Oliver J. Harrison ${ }^{\mathrm{a}, \mathrm{b}, *}$, Christopher Torrens ${ }^{\mathrm{a}}$, Kareem Salhiyyah ${ }^{\mathrm{b}}$, Amit Modi $^{\mathrm{c}}$, Narain Moorjani ${ }^{\mathrm{d}}$, \\ Paul A. Townsend ${ }^{\mathrm{e}}$, Sunil K. Ohrib and Felino Cagampang ${ }^{\mathrm{a}}$
}

a Institute of Developmental Sciences, Faculty of Medicine, University of Southampton, Southampton, UK

b Department of Cardiac Surgery, University Hospital Southampton, Southampton, UK

c Sussex Cardiac Centre, Brighton, UK

${ }^{d}$ Department of Cardiac Surgery, Royal Papworth Hospital, University of Cambridge, Cambridge, UK

e Division of Cancer Sciences, Faculty of Biology, Medicine and Health, Manchester Cancer Research Centre, Manchester Academic Health Science Centre, University of Manchester, Manchester, UK

* Corresponding author. Department of Cardiac Surgery, D-level, North Wing (MP 46), Southampton General Hospital, Tremona Road, Southampton SO16 6YD, UK. Tel: +44-23-81204938; fax: +44-23-81204526; e-mail: ojharrison@doctors.org.uk (O.J. Harrison).

Received 14 August 2018; received in revised form 2 December 2018; accepted 10 December 2018
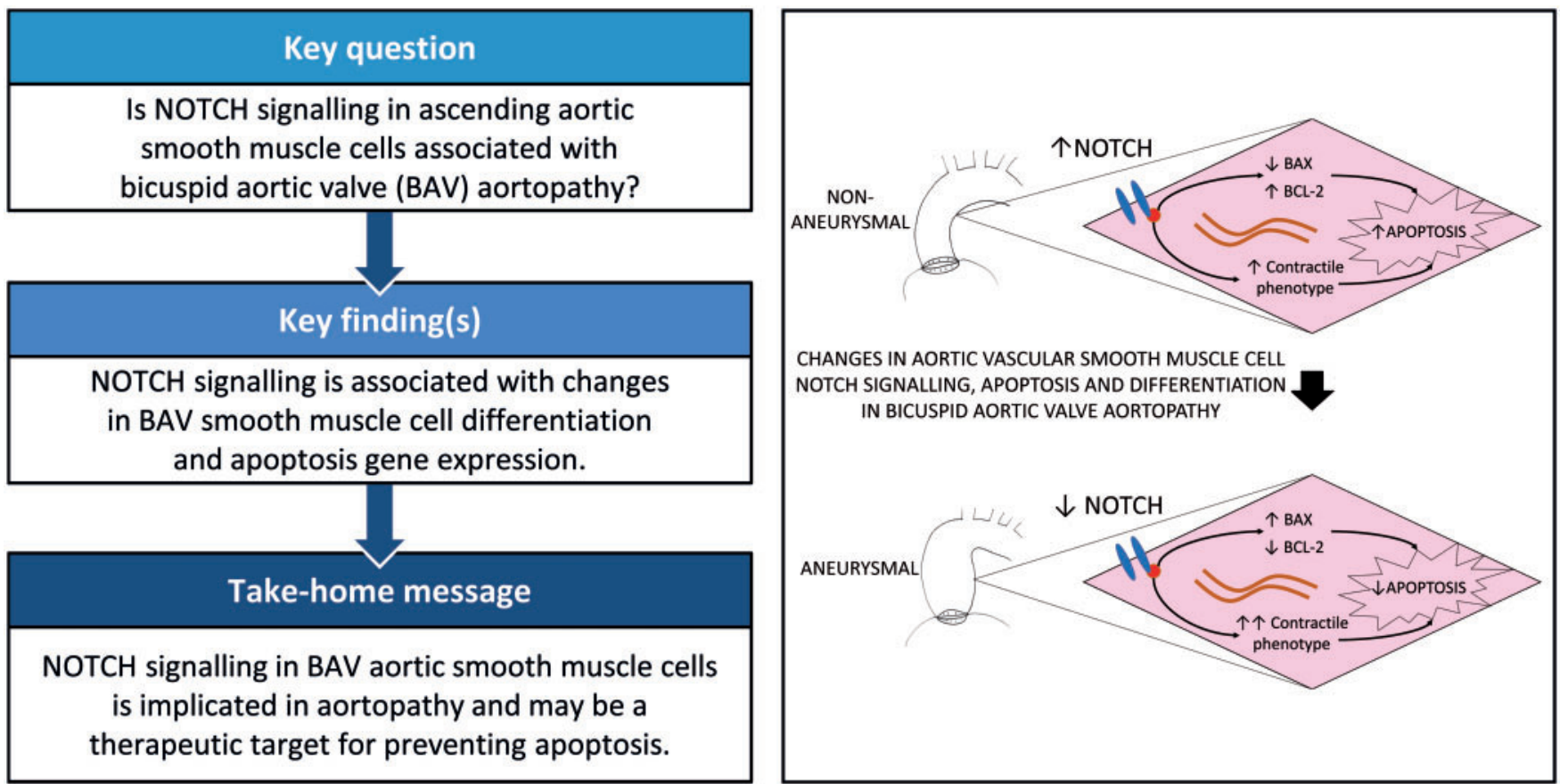


\begin{abstract}
OBJECTIVES: Bicuspid aortic valve disease is common and is associated with ascending aortic aneurysms. Vascular smooth muscle cell (VSMC) apoptosis is characteristic of the ascending aorta of bicuspid patients, and NOTCH1 gene mutations have also been linked to the disease. NOTCH signalling is a fundamental cell signalling pathway, which dictates cell fate decisions including apoptosis. Our objective was to elucidate the role of NOTCH signalling in VSMC apoptosis and differentiation in bicuspid aortopathy.
\end{abstract}

METHODS: Ascending aortic biopsies were obtained from 19 bicuspid and 12 tricuspid aortic valve patients and were sub-classified into 4 groups according to the maximum ascending aortic diameter (aneurysmal $\geq 45 \mathrm{~mm}$ ). Apoptotic VSMCs were counted by light microscopy using a TUNEL assay. Gene expression of key regulators of NOTCH signalling (NOTCH1 and HES1), apoptosis (BAX and BCL-2) and VSMC differentiation (MYH11, CNN1 and MYH10) were quantified using quantitative real-time PCR. Primary VSMCs were cultured from 2 tricuspid aortic valve and 2 bicuspid aortic valve patients, NOTCH signalling was inhibited with N-[N-(3,5-Difluorophenacetyl)-I-alanyl]-Sphenylglycine t-butyl ester, and the gene expression was again quantified.

RESULTS: The apoptotic cell count was significantly higher in bicuspid aortic valve patients (3.2 cells $/ 50000 \mu \mathrm{m}^{2} \mathrm{vs} 1.1$ cells/50 $000 \mu \mathrm{m}^{2}$; $P=0.033$ ). There was a trend towards lower apoptotic cell count in the aneurysmal versus non-aneurysmal tricuspid and bicuspid groups and an increased ratio of proapoptotic gene expression, which was not statistically significant. This was associated with a 2.8 -fold increase in contractile gene expression $(P=0.026)$ and a 2.0 -fold increase in NOTCH signalling gene expression in bicuspid versus tricuspid aortic valve patients $(P=0.022)$. NOTCH inhibition in cultured VSMCs induced a similar pattern of increased proapoptotic and procontractile gene expressions.

CONCLUSIONS: This preliminary study suggests that NOTCH activation in the non-aneurysmal bicuspid aortas may underlie aortopathy by influencing VSMC apoptosis and differentiation. NOTCH signalling manipulation may provide a therapeutic target for preventing aneurysms in bicuspid patients. Further studies with larger sample sizes are needed to substantiate the present findings.

Keywords: Bicuspid aortic valve • Ascending aortic aneurysm • Vascular smooth muscle cell • NOTCH signalling • Apoptosis • Cell differentiation

\section{INTRODUCTION}

Bicuspid aortic valve (BAV) disease is the most common congenital cardiac anomaly affecting between $1-2 \%$ of the population with a male predominance of $3: 1$ [1-3]. It results when only 2 of the 3 aortic valve leaflets form and is associated with accelerated aortic valve degeneration and the need for valve replacement [4]. BAV is also a major risk factor for ascending aortic aneurysms, which affects approximately $50 \%$ of patients during their lifetime $[5,6]$. Left undetected, aneurysms may rupture or dissect, which is frequently fatal. Despite a growing research interest, the mechanisms underlying this association remain to be elucidated.

Microscopic examination of the BAV ascending aortic media reveals medial necrosis, fibrillin degradation, elastin fragmentation and vascular smooth muscle cell (VSMC) apoptosis [7]. VSMC apoptosis is seen in BAV aortas before aortic dilation occurs, suggesting that a pre-existing genetic defect may exist [8] One of the few genetic mutations identified in BAV populations are those in the human gene Notch homolog 1, translocationassociated (Drosophila), also known as NOTCH1, an evolutionarily conserved cell signalling mechanism that dictates cell fate decisions $[9,10]$. The pathway is central to the coordination of neural crest cell migration during cardiac embryogenesis. These cells go on to populate the developing ascending aorta as primitive VSMCs [11]. NOTCH signalling is also implicated in apoptosis inhibition [12] and promotion of the contractile VSMC phenotype [13]. Recently, NOTCH signalling in aortic valve endothelial cells has been demonstrated to differ in BAV and TAV patients and may induce accelerated valve calcification in BAV patients [14].

Currently, there is little evidence for defective NOTCH signalling in BAV aortopathy, and a role in VSMC apoptosis and differentiation remains to be elucidated [15]. Given the central role of NOTCH signalling in cell fate decisions and its implication in BAV disease, we hypothesized that changes in NOTCH signalling may underlie increased VSMC apoptosis in BAV aortopathy [15].
Furthermore, the influence of NOTCH signalling on cellular differentiation may underlie the failure of VSMCs to respond to and repair the degenerated extracellular matrix (ECM), which is characteristic of BAV aortopathy [7]. This preliminary study investigates the impact of NOTCH signalling on VSMC apoptosis, apoptotic gene expression and VSMC differentiation gene expression in the context of BAV aortopathy, using both aortic tissue and VSMC culture models. We hypothesize that changes in NOTCH signalling may underlie increased VSMC apoptosis and differentiation in BAV aortopathy and thus represent a key pathological pathway.

\section{MATERIALS AND METHODS}

\section{Patient selection}

Ethical approval was granted by the Hampshire B NRES committee south central (REC Ref: 11/SC/0258) to collect ascending aortic wall biopsies from patients aged 18-80years undergoing aortic valve replacement and/or ascending aortic replacement at the University Hospital Southampton (Southampton, UK). Written informed consent was provided by study participants who were approached on a consecutive basis. Biopsies were taken from the anterior aspect of the aorta from the edge of the aortotomy line. Exclusion criteria included mitral valve disease (greater than mild), atherosclerosis of the ascending aorta, infective endocarditis and known genetic conditions (e.g. Marfan syndrome). The maximum ascending aortic diameter was obtained on perioperative transoesophageal echocardiography, and an aneurysm was defined as one with a diameter $\geq 45 \mathrm{~mm}$ as per the European Society of Cardiology/European Association for Cardio-Thoracic Surgery guidelines [16]. Aortic valve morphology was defined intraoperatively by the operating surgeon and confirmed by a second surgeon as either bicuspid $(n=19)$ or 
Table 1: Characteristics of the study groups (mean \pm standard deviation or $\%$ rounded to nearest whole number)

\begin{tabular}{|c|c|c|c|c|c|}
\hline & $\mathrm{TU}(n=7)$ & $\operatorname{TD}(n=5)$ & $\mathrm{BU}(n=9)$ & $\mathrm{BD}(n=10)$ & $P$-value \\
\hline Age (years) & $68.6 \pm 7.9$ & $69.8 \pm 5.5$ & $58.8 \pm 12.0$ & $54.2 \pm 10.5$ & 0.012 \\
\hline Gender (n) & & & & & 0.420 \\
\hline Male & 5 & 3 & 4 & 8 & \\
\hline Female & 2 & 2 & 5 & 2 & \\
\hline Body mass index $\left(\mathrm{kg} / \mathrm{m}^{2}\right)$ & $28.9 \pm 4.2$ & $27.2 \pm 2.8$ & $30.4 \pm 4.6$ & $29.9 \pm 4.5$ & 0.581 \\
\hline Operation (n) & & & & & 0.013 \\
\hline AVR & 6 & 0 & 6 & 0 & \\
\hline ARR & 0 & 1 & 0 & 2 & \\
\hline AAR & 0 & 0 & 0 & 1 & \\
\hline AVR and ARR & 0 & 1 & 0 & 1 & \\
\hline AVR and AAR & 0 & 1 & 1 & 4 & \\
\hline ARR and AAR & 0 & 2 & 0 & 0 & \\
\hline AVR, ARR and AAR & 0 & 0 & 1 & 2 & \\
\hline AVR + other aortic procedures & 1 & 0 & 1 & 0 & \\
\hline \multicolumn{6}{|l|}{ Admission blood pressure $(\mathrm{mmHg})$} \\
\hline Systolic & $142 \pm 23$ & $142 \pm 11$ & $138 \pm 14$ & $16 \pm 16$ & 0.841 \\
\hline Diastolic & $74 \pm 9$ & $80 \pm 10$ & $75 \pm 10$ & $76 \pm 13$ & 0.797 \\
\hline Smoking status (n) & & & & & 0.836 \\
\hline Non-smoker & 2 & 2 & 5 & 4 & \\
\hline Ex-smoker & 4 & 2 & 4 & 4 & \\
\hline Current smoker & 1 & 1 & 0 & 2 & \\
\hline Type 2 diabetes ( $n$ ) & 3 & 0 & 2 & 1 & 0.232 \\
\hline Aortic valve disease $(n)$ & & & & & 0.040 \\
\hline Normal & 0 & 1 & 0 & 1 & \\
\hline Isolated stenosis & 4 & 0 & 4 & 1 & \\
\hline Moderate & 0 & 0 & 0 & 0 & \\
\hline Severe & 4 & 0 & 4 & 1 & \\
\hline Isolated regurgitation & 1 & 4 & 2 & 3 & \\
\hline Moderate & 0 & 1 & 0 & 0 & \\
\hline Severe & 1 & 3 & 2 & 3 & \\
\hline Mixed & 2 & 0 & 3 & 5 & \\
\hline Left ventricular ejection fraction (\%) (n) & & & & & 0.017 \\
\hline $45-70$ & 7 & 3 & 9 & 8 & \\
\hline $35-44$ & 0 & 2 & 0 & 0 & \\
\hline$<35$ & 0 & 0 & 0 & 0 & \\
\hline Unknown & 0 & 0 & 0 & 2 & \\
\hline
\end{tabular}

Bold text indicates $P$-values that are statistically significant.

$P$-values are given for difference between the groups for each demographic.

AAR: ascending aortic replacement; ARR: aortic root replacement; AVR: aortic valve replacement; BD: bicuspid aortic valve, aneurysmal; BU: bicuspid aortic valve, non-aneurysmal; TD: tricuspid aortic valve, aneurysmal; TU: tricuspid aortic valve, non-aneurysmal.

tricuspid $(n=12)$. Subsequently, patients were divided into 4 groups: BAV with an dilated/aneurysmal aorta or the undilated/ non-aneurysmal aorta and tricuspid aortic valve (TAV) with an dilated/aneurysmal aorta or an undilated/non-aneurysmal aorta. All morphological assessments concurred with preoperative transoesophageal echocardiography images, which were reviewed by a consultant cardiologist. Because of the limited time scale for recruitment, the maximum possible number of patients were enrolled. More BAV patients underwent surgery during the study period, therefore the study group numbers differed. The mean age of the patients was $61.3( \pm 11.4)$, and $65 \%$ of the patients $(n=20)$ were men. An ascending aortic aneurysm was present in 10 of 19 BAV and 5 of 12 TAV patients (Table 1).

\section{Quantification of apoptotic vascular smooth muscle cells in the aortic media}

Aortic biopsies were snap-frozen in Optimum Cutting Temperature (OCT; Agar Scientific, UK) medium, sectioned at $7 \mu \mathrm{m}$ and mounted on microscopy slides. The TACS TdT In Situ Apoptosis Detection Kit (Trivigen, USA) was used to indicate cell apoptosis according to the standard protocol (Supplementary
Material, File S1). Additional control measures included a positive control using a nuclease step (creating double-stranded DNA breaks seen in apoptotic cells) and a negative control, which excluded the labelling mix. All samples were stained in duplicate. Each slide was imaged using a dotSlide Virtual Slide System (Olympus, UK). Ten subfields $\left(50000 \mu \mathrm{m}^{2}\right)$ were scanned at $40 \times$ magnification, and a macro was written for the Fiji-ImageJ image analysis software [17] to count the viable and apoptotic cells. The apoptotic index was calculated as follows:

$$
\text { Apoptotic index }=\frac{\text { Number of apoptotic nuclei }}{\text { Total number of nuclei }} \times 100
$$

\section{Expression of NOTCH signalling, apoptosis and vascular smooth muscle cell differentiation genes in aortic tissue}

RNA extraction. Snap-frozen aortic samples were crushed in liquid nitrogen prior to RNA extraction as previously detailed by 
Table 2: Demographics and culture data of the patients used for NOTCH signalling inhibition in vascular smooth muscle cells

\begin{tabular}{lllll}
\hline Number of patient & 1 & 2 & 3 & 4 \\
\hline Age (years) & 66 & 59 & 63 & 71 \\
Gender & Female & Male & Male & Male \\
Aortic valve morphology & Tricuspid & Bicuspid & Bicuspid & Tricuspid \\
Max aortic diameter (mm) & 49 & 54 & 57 & 27 \\
Time to $\geq 80 \%$ confluence (days) & 28 & 37 & 31 & 606000 \\
Cell count at first passage & 1005000 & 206400 & P2 & 808644 \\
Passage at which cells were used & P2 & P3 & & P2 \\
\hline
\end{tabular}

our group using the spin column-based Qiagen RNeasy Fibrous kit (Qiagen, USA) as per protocol [18]. RNA yield and quality were confirmed using a NanoDrop spectrophotometer (ThermoFisher Scientific, UK) and gel electrophoresis. RNA integrity was assured using the Agilent Bioanalyser (Agilent Technologies, USA) with the mean RNA integrity (RIN) values of $8.8( \pm 1.1)$. RNA was reverse transcribed using the GoScript Reverse Transcription Kit (Promega, UK).

Quantitative real-time PCR. Primer sequences for the genes of interest can be found in the Supplementary Material, Table S1. Gene expression was measured using quantitative real-time PCR (Supplementary Material, File S2).

\section{Inhibition of NOTCH signalling in vascular smooth muscle cells}

Vascular smooth muscle cell culture. Primary VSMCs were raised from 4 of the aortic explants used in the aortic tissue experiments ( $n=4$; Table 2). A detailed description of the method is found in the Supplementary Material, File S3. VSMCs were seen to grow out from aortic explants in a characteristic 'hills and valleys' pattern and demonstrated immunofluorescence for $\alpha$ smooth muscle actin.

Inhibition of NOTCH signalling. N-[N-(3,5-Difluorophenacetyl)-I-alanyl]-S-phenylglycine t-butyl ester (DAPT) is a $\gamma$ secretase inhibitor and an indirect inhibitor of NOTCH signalling (Supplementary Material, File S4) [19]. DAPT concentration and time points were optimized in preliminary experiments. DAPT (dissolved in dimethyl sulfoxide (DMSO)) at $1 \mu \mathrm{M}$ was used together with $\mathrm{DMSO} /$ medium only control. Culture wells were seeded with 100000 cells and performed in duplicate. Cells were incubated for $48 \mathrm{~h}$ at $37^{\circ} \mathrm{C}$ with $5 \% \mathrm{CO}_{2}$ prior to adding DAPT solutions. Baseline cultures were harvested, and experimental solutions were added. Further harvests were performed at $6 \mathrm{~h}$ and $12 \mathrm{~h}$. Relative gene expression of NOTCH1, HES1, BAX, BCL-2, MYH11, CNN1 and MYH10 was calculated using quantitative real-time PCR as described above. SDHA and YWAZ were identified as stable reference genes (unpublished data).

\section{Statistical analysis}

Statistical analysis was performed using the IBM SPSS statistics package (v24) and the GraphPad Prism (v7). One TAV patient and 3 BAV patients had inadequate biopsy mass to perform cell counting, and 2 BAV patients had inadequate tissue to perform gene expression. Therefore, cell counts were performed on 11 TAV and 16 BAV samples, and gene expression was performed on 12 TAV and 17 BAV patients. $P$-values for statistical significance were calculated using the $t$-test and the analysis of variance (ANOVA) for continuous variables and the $\chi^{2}$ test for categorical variables, where Yates' correction was performed if minimum counts were not met. The one-way ANOVA was used for aortic gene expression data, and 2-way ANOVA with the Tukey post hoc test was used for VSMC gene expression data. Linear regression excluded any interaction between valve morphology and the aortic diameter such that each could be independently compared. Normality testing was performed on all outcome variables using visual assessment and the Shapiro-Wilk test. Logarithmic transformation was performed prior to statistical testing where outcome variables were not normally distributed but are presented untransformed. A $P$-value of $<0.05$ was considered statistically significant. Gene expression values are in arbitrary units relative to reference genes and conveyed as foldchanges. For the VSMC experiments, the fold-change in gene expression compared to the baseline is quoted. The maximum possible number of samples were obtained within the timescale of the project. However, a retrospective power calculation based on $\alpha$ (type I error) of 0.05 and power of 0.8 suggested a sample size of 20 per group.

\section{RESULTS}

\section{Quantification of apoptotic vascular smooth muscle cells in the aortic media}

Apoptotic cell count was significantly higher in BAV versus TAV patients (3.2 cells $/ 50000 \mu \mathrm{m}^{2}$ vs 1.1 cells $/ 50000 \mu \mathrm{m}^{2} ; P=0.033$ ), in the absence of any significant difference in the viable cell count (23.0 cells $/ 50000 \mu \mathrm{m}^{2}$ vs 19.0 cells $/ 50000 \mu \mathrm{m}^{2} ; P=0.442 ;$ Fig. $1 \mathrm{~A}$ and $\mathrm{B})$. Although the apoptotic index was 1.5 -fold higher in the BAV group, this was not statistically significant ( $P=0.299$; Fig. $1 C)$. There was no significant difference in either the viable or the apoptotic cell count between TAV and BAV patients with aneurysmal versus non-aneurysmal aortas (Fig. 1D and E). However, there was a trend towards decreased apoptotic cell count in the aneurysmal versus the non-aneurysmal TAV and BAV aortas ( 0.3 cells/ $50000 \mu \mathrm{m}^{2}$ vs 1.6 cells/50 $000 \mu \mathrm{m}^{2}$ and 2.2 cells/50 $000 \mu \mathrm{m}^{2}$ vs 4.4 cells $/ 50000 \mu \mathrm{m}^{2}$, respectively; $P=0.087$ ). The apoptotic index followed a similar trend between aneurysmal and non-aneurysmal aortas, but this was not statistically significant (Fig. 1F). 

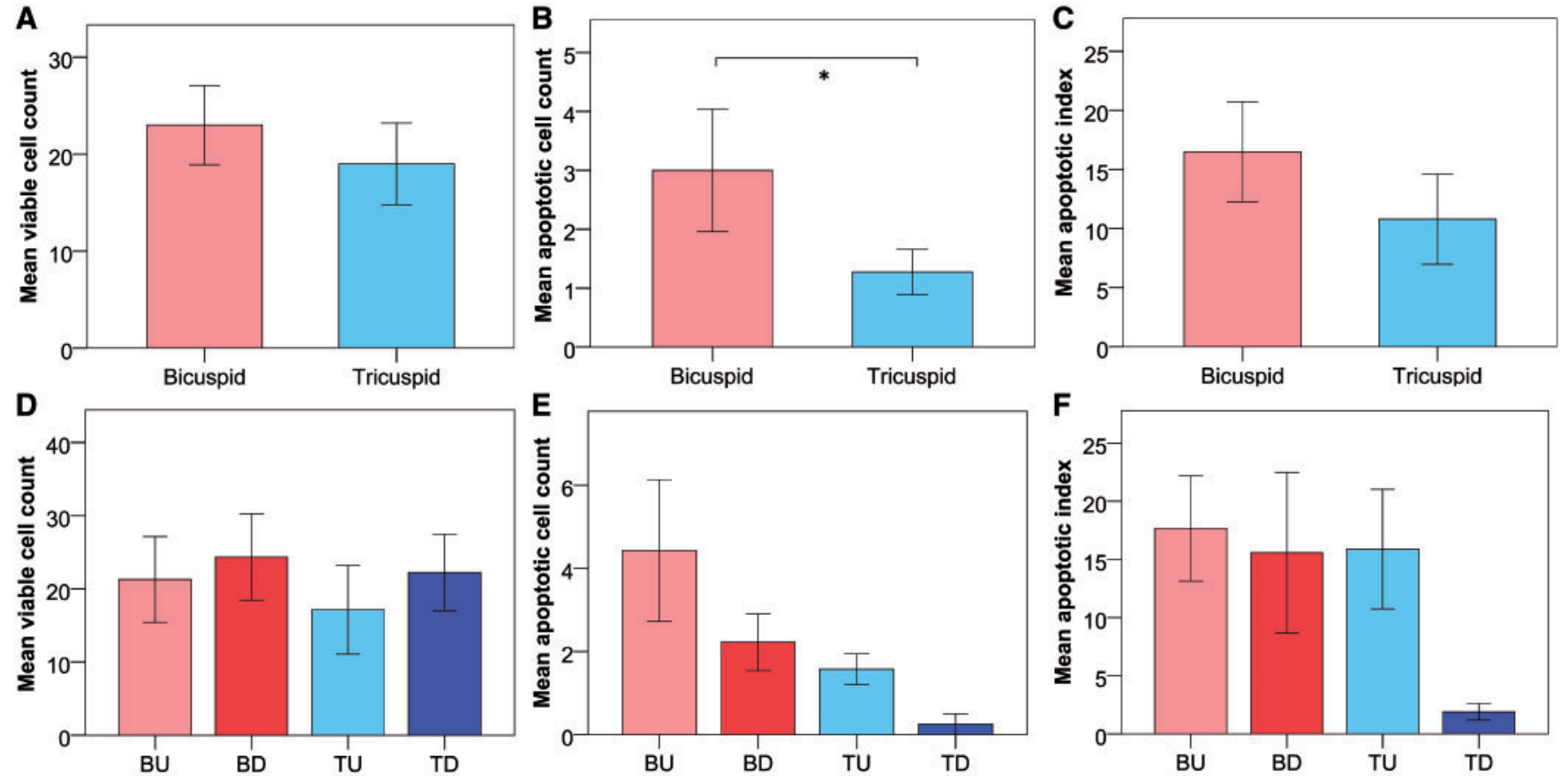

Figure 1: TUNEL cell counts by valve morphology and maximum ascending aortic diameter. (A and D) Mean viable cell count/50 $000 \mu m^{2}$. (B and E) Mean apoptotic cell count $/ 50000 \mathrm{\mu m}^{2}$. (C and F) Mean apoptotic index. BU $(n=7)$ and BD $(n=9)$ aortas, and TU $(n=7)$ and TD $(n=4)$ aortas. Error bars $= \pm 1$ standard error (SE). ${ }^{*} P<0.05$. BD: bicuspid aortic valve patients with aneurysmal aortas; BU: bicuspid aortic valve patients with non-aneurysmal aortas; TD: tricuspid aortic valve patients with aneurysmal aortas; TU: tricuspid aortic valve patients with non-aneurysmal aortas.
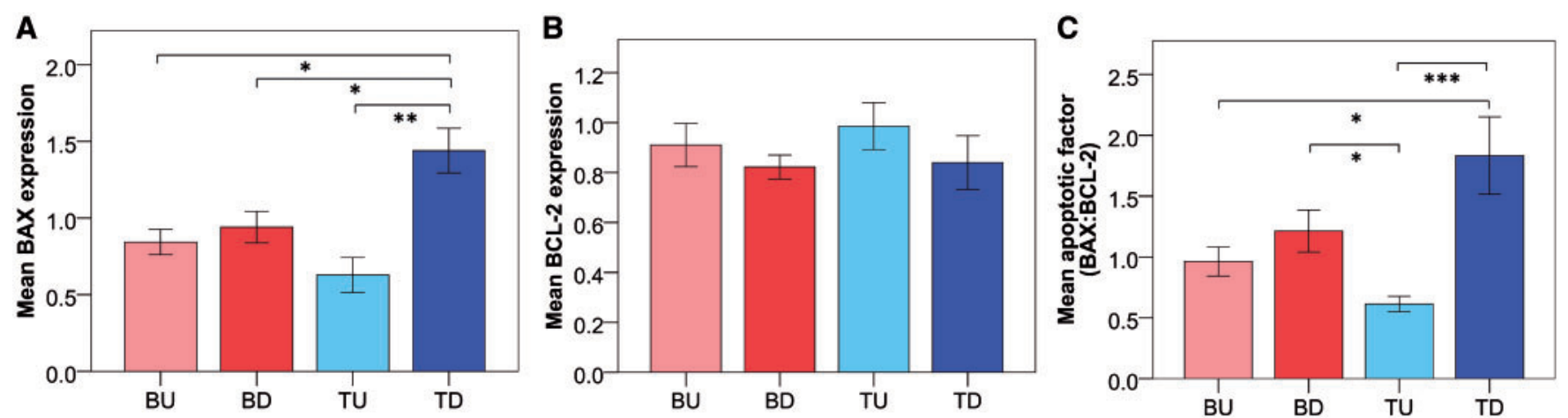

Figure 2: Ascending aortic apoptotic gene expression in bicuspid aortic valve and tricuspid aortic valve patients by aortic dimension. (A) Mean $B A X$ expression; (B) mean BCL-2 expression; and (C) mean apoptotic factor (BAX:BCL-2 ratio). BU $(n=7)$ and BD $(n=10)$ aortas, and TU $(n=7)$ and TD $(n=5)$ aortas. Expression relative to reference genes (GAPDH and UBC). Error bars $= \pm 1$ SE. ${ }^{*} P<0.05,{ }^{* *} P<0.01$ and ${ }^{* * *} P<0.001$. BD: bicuspid aortic valve patients with aneurysmal aortas; BU: bicuspid aortic valve patients with non-aneurysmal aortas; TD: tricuspid aortic valve patients with aneurysmal aortas; TU: tricuspid aortic valve patients with non-aneurysmal aortas.

A comparison between aneurysm and non-aneurysm groups are seen in the Supplementary Material, Fig. S1.

\section{Expression of NOTCH signalling, apoptosis and vascular smooth muscle cell differentiation genes in aortic tissue}

Apoptotic gene expression. Expression differences in proapoptotic BAX and anti-apoptotic BCL-2 genes between BAV and TAV patients with the non-aneurysmal and aneurysmal aortas were calculated. A ratio between BAX and BCL-2 (apoptotic factor) was calculated as a measure of tendency towards apoptosis as has been used previously [20]. BAX expression was significantly higher in aneurysmal TAV patients compared to non-aneurysmal TAV patients (2.3-fold, $P=0.001)$, non-aneurysmal BAV patients (1.7-fold, $P=0.011$ ) and aneurysmal BAV patients (1.5-fold, $P=0.025$; Fig. 2A). $B C L-2$ expression was lower in the aneurysmal versus the non-aneurysmal aortas (0.9-fold for both TAV and $\mathrm{BAV}$ ), but this was not statistically significant (Fig. 2B). The apoptotic factor (BAX:BCL-2 ratio) reflected these observations (Fig. 2C).

Vascular smooth muscle cell differentiation gene expression. Given the paradoxical association between apoptotic gene expression and apoptotic cell count in aneurysmal versus non-aneurysmal aortas, it was hypothesized that differences in VSMC differentiation may occur in parallel. The expression of contractile phenotype genes MYH11 and CNN1, together with the 

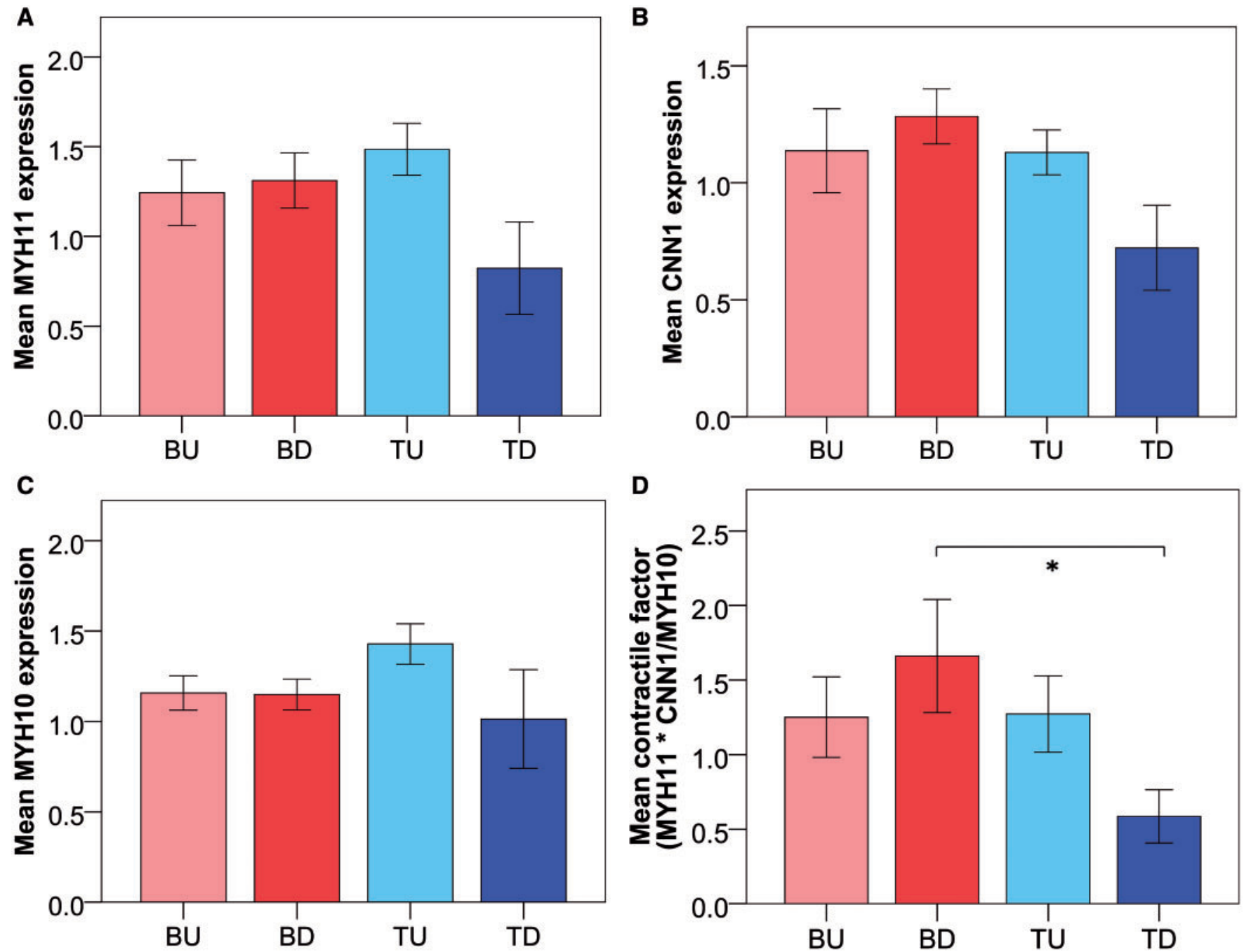

Figure 3: Ascending aortic vascular smooth muscle cell differentiation gene expression in bicuspid aortic valve and tricuspid aortic valve patients by aortic dimension. (A) Mean MYH11 expression; (B) mean CNN1 expression; (C) mean MYH10 expression; (D) mean contractile factor (MYH17xCNN1:MYH10 ratio). BU ( $n=7)$ and BD $(n=10)$ aortas, and TU $(n=7)$ and TD $(n=5)$ aortas. Expression relative to reference genes (GAPDH and UBC). Error bars $= \pm 1$ SE. ${ }^{*} P<0.05$. BD: bicuspid aortic valve patients with aneurysmal aortas; BU: bicuspid aortic valve patients with non-aneurysmal aortas; TD: tricuspid aortic valve patients with aneurysmal aortas; TU: tricuspid aortic valve patients with non-aneurysmal aortas.

synthetic phenotype gene MYH10, was calculated [21]. Expression of all 3 VSMC differentiation genes tended to be higher in the aneurysmal BAV aortas and lower in the aneurysmal TAV aortas compared to their non-aneurysmal equivalents, but these were not statistically significant (Fig. 3A-C). The contractile factor (MYH17xCNN1:MYH10) was significantly higher by 2.8-fold in aneurysmal BAV versus aneurysmal TAV patients ( $P=0.026$; Fig. 3D). Furthermore, the contractile factor was 1.3-fold higher in the aneurysmal versus the non-aneurysmal BAV aortas and 0.5-fold lower in the aneurysmal versus the non-aneurysmal TAV aortas, but this was not found to be statistically significant.

NOTCH signalling gene expression. It was hypothesized that the differences in apoptosis and differentiation gene expression may be linked to NOTCH signalling activation. To elucidate this, the expression of NOTCH1 and its downstream target HES1 were calculated. There was no significant difference in NOTCH1 expression between BAV and TAV patients of differing aortic dimensions (Fig. 4A). However, HES1 expression was significantly higher in BAV patients with non-aneurysmal aortas by 2.0 -fold versus their TAV counterparts ( $P=0.022$; Fig. 4B). HES1 expression was 0.7-fold lower in the aneurysmal BAV group versus the non-aneurysmal group, but this was not found to be statistically significant. A reciprocal trend towards increased HES1 expression by 2.0 -fold in aneurysmal versus non-aneurysmal TAV patients was also seen, but again this was not found to be statistically significant.

\section{Inhibition of NOTCH signalling in vascular smooth muscle cells}

To investigate whether gene expression changes could be reproduced in vitro, NOTCH signalling was inhibited in primary VSMC cultures with DAPT. At $6 \mathrm{~h}$ and $12 \mathrm{~h}, \mathrm{HES} 1$ expression was significantly reduced by 0.4 -fold in the BAV DAPT-treated cells versus control $(P<0.05)$, an effect that was not replicated in the TAV DAPT-treated cells (Fig. 5A). The apoptotic factor demonstrated significant decreases from the baseline in both TAV control cells by 0.6 -fold $(P<0.05)$ and TAV DAPT cells by 0.4 -fold $(P<0.01)$ at $6 \mathrm{~h}$ (Fig. 5B). A similar trend was seen in the BAV cells at $6 \mathrm{~h}$, but 

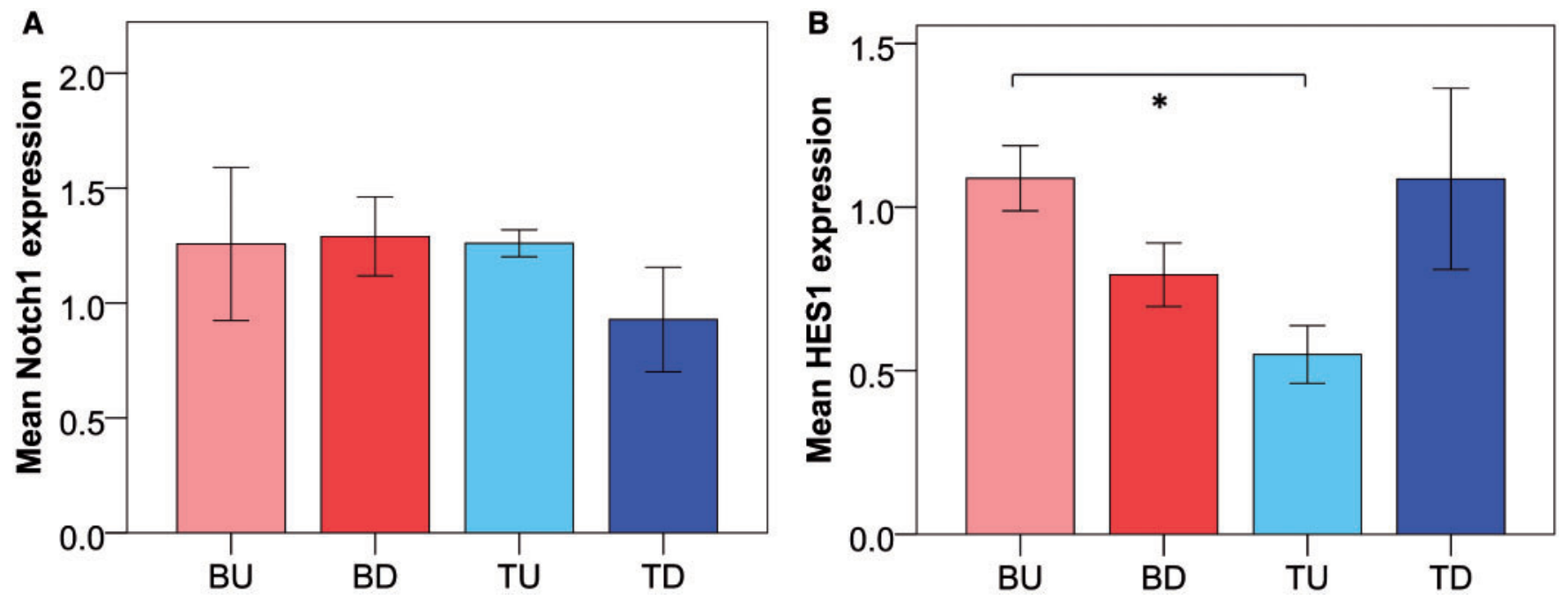

Figure 4: NOTCH signalling gene expression in the ascending aortas of bicuspid aortic valve and tricuspid aortic valve patients by aortic dimension. (A) Mean NOTCH1 expression and (B) mean HES1 expression. BU $(n=7)$ and BD $(n=10)$ aortas, and TU $(n=7)$ and TD $(n=5)$ aortas. Expression relative to reference genes $\left(G A P D H\right.$ and UBC). Error bars $= \pm 1$ SE. ${ }^{*}<<0.05$. BD: bicuspid aortic valve patients with aneurysmal aortas; $B U$ : bicuspid aortic valve patients with non-aneurysmal aortas; TD: tricuspid aortic valve patients with aneurysmal aortas; TU: tricuspid aortic valve patients with non-aneurysmal aortas.

A

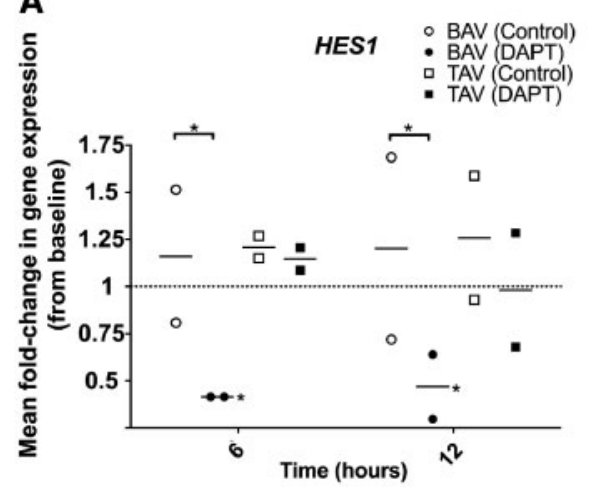

B

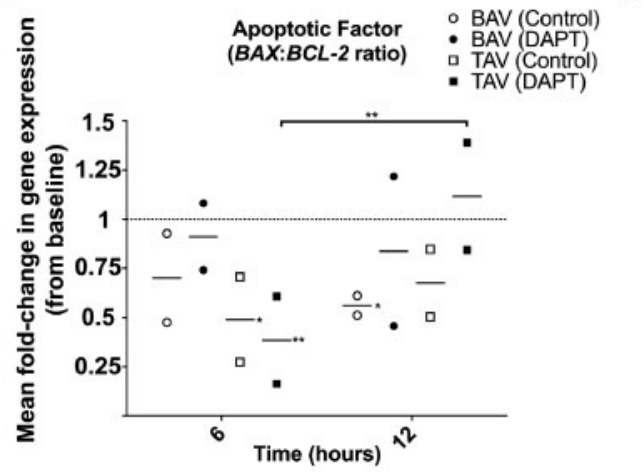

C

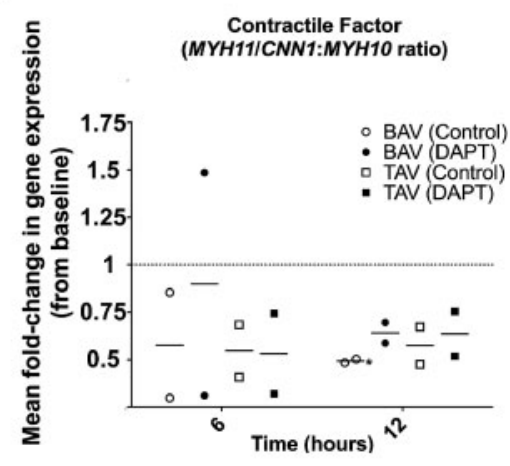

Figure 5: Fold-change in NOTCH signalling, apoptosis and vascular smooth muscle cell differentiation gene expression compared to baseline at $6 \mathrm{~h}$ and $12 \mathrm{~h}$ following inhibition of NOTCH signalling with DAPT in primary vascular smooth muscle cell cultures. (A) HES1 gene expression changes. (B) Apoptotic factor (BAX:BCL-2 ratio) changes. (C) Contractile factor (MYH17XCNN1:MYH10 ratio) changes. Expressions relative to reference genes (SDHA and YWHAZ). N=2 in each experimental group. Bars represent the mean change in expression compared to baseline at time $=0 \mathrm{~h} .{ }^{*} P<0.05$ and ${ }^{*} P<0.01$. BAV: bicuspid aortic valve; DAPT: $\mathrm{N}$-[N-(3,5Difluorophenacetyl)-I-alanyl]-S-phenylglycine t-butyl ester; TAV: tricuspid aortic valve.

this was not found to be statistically significant. Nevertheless, NOTCH inhibition with DAPT attenuated this decrease compared to the BAV control. A similar pattern was seen at $12 \mathrm{~h}$; however, the apoptotic factor increased in the TAV DAPT cells by 1.1-fold compared to baseline and decreased in the TAV control by 0.6 fold compared to baseline, but this was not significant. The contractile factor was significantly reduced by 0.5 -fold compared to baseline in the BAV control cells at $12 \mathrm{~h}(P<0.05)$, and there was a trend towards reduction in the BAV DAPT cells by 0.7 -fold, although this was not statistically significant (Fig. 5C). This trend was reflected in the BAV control and BAV DAPT cells at $6 \mathrm{~h}$ but again was not statistically significant.

\section{DISCUSSION}

Increased VSMC apoptosis in the ascending aorta of patients with BAV disease is well documented, but the underlying mechanisms, particularly the contribution of VSMC differentiation state and NOTCH signalling has yet to be elucidated. This study demonstrated an inverse relationship between observed VSMC apoptosis and apoptotic gene expression in BAV aortopathy, which was associated with contractile VSMC differentiation. NOTCH signalling activation demonstrated a similar association, which was confirmed in vitro where $\mathrm{NOTCH}$ inhibition promoted the contractile phenotype and proapoptotic gene expression. These preliminary findings suggest that defective NOTCH signalling may underlie the pathophysiology of BAV aortopathy and represents an area for further research.

Increased VSMC apoptosis is a hallmark of BAV aortopathy, but the molecular mechanisms are poorly understood [22]. This study demonstrated that in aneurysmal aortas, the number of cells undergoing apoptosis tends to decrease in both BAV and TAV patients, which is consistent with previous reports [22]. However, a paradoxical increase in the $B A X: B C L-2$ ratio was also seen implying that VSMCs may develop resistance to apoptosis. 
Previous studies suggest that the synthetic VSMC phenotype may confer resistance to apoptosis [23]. The present results support this, demonstrating lower contractile gene expression associated with higher apoptotic gene expression and lower apoptotic cell count in the TAV group. Conversely, VSMCs in aneurysmal BAV aortas demonstrated a higher contractile gene expression, suggesting increased sensitivity to apoptosis, which may contribute to aortopathy.

$\mathrm{NOTCH}$ may play a role in any such changes in VSMC differentiation; however, this is disputed $[24,25]$. NOTCH1 is an important gene in BAV disease, and our hypothesis was that changes in NOTCH signalling may contribute to BAV aortopathy. Significantly increased HES1 expression (a surrogate marker of NOTCH activation) in non-aneurysmal BAV aortas versus nonaneurysmal TAV aortas was demonstrated with a paradoxical decrease in BAV and increase in TAV patients with an aneurysm. A recent study by Balistreri et al. [26] similarly concluded that $\mathrm{NOTCH}$ activation increases in aneurysmal versus nonaneurysmal TAV aortas and aneurysmal TAV versus aneurysmal BAV aortas. One possible explanation is that infiltrating inflammatory cells (characteristic of TAV aneurysms) expressing NOTCH ligands increase VSMC NOTCH activation [27]. Similarly, inhibition of NOTCH signalling in abdominal aortic aneurysms (also mediated by inflammation) reduces the aortic diameter in a mouse model [28].

Another important observation from this study was that inhibiting NOTCH signalling with DAPT significantly reduced HES1 expression in BAV VSMCs but did not affect HES1 expression in TAV VSMCs. As is suggested by our results, a defect in activation of NOTCH signalling in BAV VSMCs may be present, for example, a difference in the activity of $\gamma$-secretase or its affinity for DAPT. Alternatively, there may be reduced expression of NOTCH ligands in BAV VSMCs as previously demonstrated by Sciacca et al. [29]. Reduced ligand expression in culture may lead to reduced NOTCH receptor activation and HES1 expression, and a possible compensatory upregulation in NOTCH receptor transcription.

\section{Limitations}

This study presents a number of limitations. The limited sample size may have resulted in the lack of statistical significance in some of the comparisons and several hypotheses rely on trends, which will require further validation. Differing shear stresses on the aortic wall may affect gene expression. Although samples were taken from the same area, we cannot exclude an effect of shear stresses on gene expression. Furthermore, the data would have been strengthened if validated at the protein level. Any mismatch between transcription and translation could result in significant mRNA changes without a change in the biologically active proteins. An attempt was made to correlate aortic and VSMC gene expression. There appeared to be some congruency between gene expressions according to valve morphology, but this was not statistically significant. Similarly, HES1 expression was assumed to correlate with NOTCH activation; however, this is an indirect indicator and may be influenced by other pathways. Additionally, using 2 inhibitors of NOTCH signalling would have greatly strengthened our conclusions. Furthermore, activation of other $\mathrm{NOTCH}$ receptors 2, 3 and 4 may influence the results but was not detected by our methodology. Finally, all samples used in the VSMC culture experiments were taken from aneurysmal aortas and, therefore, may not be representative of findings in non-aneurysmal aortas. Several unsuccessful attempts were made to culture VSMCs from non-aneurysmal samples, likely due to limited tissue mass.

\section{CONCLUSION}

A definitive conclusion in this preliminary study is difficult, but the results strongly suggest that inhibition of NOTCH signalling in BAV VSMCs promotes the contractile VSMC phenotype and proapoptotic gene expression. Previous studies have proposed that $\mathrm{NOTCH}$ signalling activation reduces the apoptotic drive and promotes the synthetic phenotype in VSMCs $[12,30]$. We postulate that NOTCH signalling may be central to BAV aortopathy by directly increasing apoptosis and promoting the contractile VSMC phenotype, which further sensitizes VSMCs to apoptosis.

The complex mechanisms underlying BAV aortopathy have afforded extensive research to date and will undoubtedly continue to be the focus of intense future investigations. This preliminary study has provided some insight into the role of VSMC apoptosis and differentiation in BAV aortopathy and described the important interaction of $\mathrm{NOTCH}$ signalling within these mechanisms. NOTCH signalling has been identified as an important pathway in BAV disease and may represent a future therapeutic target for preventing the progression of BAV aortopathy. Further studies are needed to substantiate these findings.

\section{SUPPLEMENTARY MATERIAL}

Supplementary material is available at EJCTS online.

\section{ACKNOWLEDGEMENTS}

The authors acknowledge Kerry Hyde and Melissa Doherty (University of Southampton) for their support in the laboratory; Claire Simner, Emma Lofthouse and Jane Cleal (University of Southampton) for assisting with optimizing, acquiring and analysing qRT-PCR data; Jon Ward and Susan Wilson for their guidance with immunohistochemistry (University of Southampton); David Chatelet (University of Southampton) for guidance on image analysis and for writing the cell-counting macros; Melanie Jannaway (University of South Florida, USA) for guidance on optimizing VSMC culture; Ella Baker and Elizabeth Miles (University of Southampton) for assistance with cell culture; and Clive Osmond (University of Southampton) for overseeing the statistical analysis.

\section{Funding}

This work was supported by the Wessex Heart Surgery fund.

Conflict of interest: none declared.

\section{REFERENCES}

[1] Basso C, Boschello M, Perrone C, Mecenero A, Cera A, Bicego D et al. An echocardiographic survey of primary school children for bicuspid aortic valve. Am J Cardiol 2004;93:661-3. 
[2] Tutar E, Ekici F, Atalay S, Nacar N. The prevalence of bicuspid aortic valve in newborns by echocardiographic screening. Am Heart J 2005; 150:513-15.

[3] Hoffman JIE, Kaplan S. The incidence of congenital heart disease. J Am Coll Cardiol 2002;39:1890-900.

[4] Roberts WC, Ko JM. Frequency by decades of unicuspid, bicuspid, and tricuspid aortic valves in adults having isolated aortic valve replacement for aortic stenosis, with or without associated aortic regurgitation. Circulation 2005;111:920-5.

[5] Della Corte A, Bancone C, Quarto C, Dialetto G, Covino FE, Scardone M et al. Predictors of ascending aortic dilatation with bicuspid aortic valve: a wide spectrum of disease expression. Eur J Cardiothorac Surg 2007;31: 397-404; discussion 404-5.

[6] Jackson V, Petrini J, Caidahl K, Eriksson MJ, Liska J, Eriksson P et al Bicuspid aortic valve leaflet morphology in relation to aortic root morphology: a study of 300 patients undergoing open-heart surgery. Eur J Cardiothorac Surg 2011;40:e118-24.

[7] Fedak PWM, de Sa MPL, Verma S, Nili N, Kazemian P, Butany J et al. Vascular matrix remodeling in patients with bicuspid aortic valve malformations: implications for aortic dilatation. J Thorac Cardiovasc Surg 2003;126:797-805.

[8] Bonderman D, Gharehbaghi-Schnell E, Wollenek G, Maurer G, Baumgartner $\mathrm{H}$, Lang IM. Mechanisms underlying aortic dilatation in congenital aortic valve malformation. Circulation 1999;99:2138-43.

[9] Artavanis-Tsakonas S, Rand MD, Lake RJ. Notch signaling: cell fate control and signal integration in development. Science 1999;284:770-6.

[10] Garg V, Muth AN, Ransom JF, Schluterman MK, Barnes R, King IN et al. Mutations in NOTCH1 cause aortic valve disease. Nature 2005;437: 270-4.

[11] Niessen K, Karsan A. Notch signaling in cardiac development. Circ Res 2008;102:1169-81.

[12] Sweeney C, Morrow D, Birney YA, Coyle S, Hennessy C, Scheller A et al. Notch 1 and 3 receptor signaling modulates vascular smooth muscle cell growth, apoptosis, and migration via a CBF-1/RBP-Jk dependent pathway. FASEB J 2004;18:1421-3.

[13] Lin CH, Lilly B. Notch signaling governs phenotypic modulation of smooth muscle cells. Vascul Pharmacol 2014;63:88-96

[14] Kostina A, Shishkova A, Ignatieva E, Irtyuga O, Bogdanova M, Levchuk K et al. Different Notch signaling in cells from calcified bicuspid and tricuspid aortic valves. J Mol Cell Cardiol 2018;114:211-19.

[15] Harrison OJ, Visan AC, Moorjani N, Modi A, Salhiyyah K, Torrens C et al. Defective NOTCH signaling drives increased vascular smooth muscle cell apoptosis and contractile differentiation in bicuspid aortic valve aortopathy: a review of the evidence and future directions. Trends Cardiovasc Med 2018.

[16] Baumgartner H, Falk V, Bax JJ, De Bonis M, Hamm C, Holm PJ et al. 2017 ESC/EACTS guidelines for the management of valvular heart disease: the task force for the management of valvular heart disease of the European Society of Cardiology (ESC) and the European Association for CardioThoracic Surgery (EACTS). Eur Heart J 2017;38:2739-91.
[17] Schindelin J, Arganda-Carreras I, Frise E, Kaynig V, Longair M, Pietzsch T et al. Fiji: an open-source platform for biological-image analysis. Nat Methods 2012;9:676-82.

[18] Harrison OJ, Moorjani N, Torrens C, Ohri SK, Cagampang FR. Endogenous reference genes for gene expression studies on bicuspid aortic valve associated aortopathy in humans. PLoS One 2016;11: e0164329.

[19] Dovey HF, John V, Anderson JP, Chen LZ, de Saint Andrieu P, Fang LY et al. Functional gamma-secretase inhibitors reduce beta-amyloid peptide levels in brain. J Neurochem 2009;76:173-81.

[20] Mohamed SA, Misfeld M, Hanke T, Charitos El, Bullerdiek J, Belge G et al. Inhibition of caspase-3 differentially affects vascular smooth muscle cell apoptosis in the concave versus convex aortic sites in ascending aneurysms with a bicuspid aortic valve. Ann Anat 2010;192:145-50.

[21] Rensen SS, Doevendans PA, van Eys GJ. Regulation and characteristics of vascular smooth muscle cell phenotypic diversity. Neth Heart J 2007;15: 100-8.

[22] Della Corte A, Quarto C, Bancone C, Castaldo C, Di Meglio F, Nurzynska $D$ et al. Spatiotemporal patterns of smooth muscle cell changes in ascending aortic dilatation with bicuspid and tricuspid aortic valve stenosis: focus on cell-matrix signaling. J Thorac Cardiovasc Surg 2008;135: 8-18.e2.

[23] Su BY, Shontz KM, Flavahan NA, Nowicki PT. The effect of phenotype on mechanical stretch-induced vascular smooth muscle cell apoptosis. J Vasc Res 2006;43:229-37.

[24] Doi H, Iso T, Sato H, Yamazaki M, Matsui H, Tanaka T et al. Jagged1-selective notch signaling induces smooth muscle differentiation via a RBPJkappa-dependent pathway. J Biol Chem 2006;281:28555-64.

[25] Proweller A, Pear WS, Parmacek MS. Notch signaling represses myocardin-induced smooth muscle cell differentiation. J Biol Chem 2005;280:8994-9004.

[26] Balistreri CR, Crapanzano F, Schirone L, Allegra A, Pisano C, Ruvolo G et al. Deregulation of Notch1 pathway and circulating endothelial progenitor cell (EPC) number in patients with bicuspid aortic valve with and without ascending aorta aneurysm. Sci Rep 2018;8:13834.

[27] Folkersen L, Wagsater D, Paloschi V, Jackson V, Petrini J, Kurtovic S et al. Unraveling divergent gene expression profiles in bicuspid and tricuspid aortic valve patients with thoracic aortic dilatation: the ASAP study. Mol Med 2011;17:1365-73.

[28] Cheng J, Koenig SN, Kuivaniemi HS, Garg V, Hans CP. Pharmacological inhibitor of notch signaling stabilizes the progression of small abdominal aortic aneurysm in a mouse model. J Am Heart Assoc 2014;3: e001064.

[29] Sciacca S, Pilato M, Mazzoccoli G, Pazienza V, Vinciguerra M. Anti-correlation between longevity gene SirT1 and Notch signaling in ascending aorta biopsies from patients with bicuspid aortic valve disease. Heart Vessels 2013;28:268-75.

[30] Morrow D, Sweeney C, Birney YA, Cummins PM, Walls D, Redmond EM et al. Cyclic strain inhibits Notch receptor signaling in vascular smooth muscle cells in vitro. Circ Res 2005;96:567-75. 